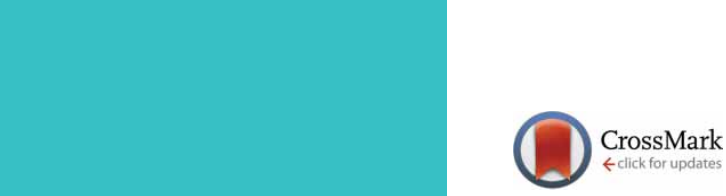

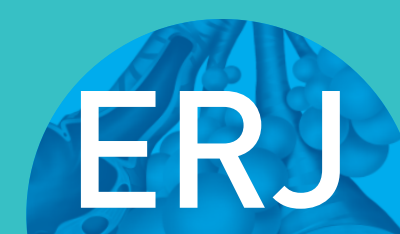

open research

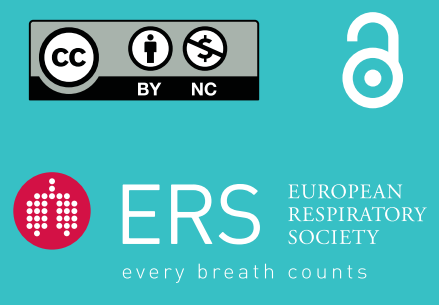

\section{European Respiratory Society International Congress, Madrid, 2019: nontuberculous mycobacterial pulmonary disease highlights}

\author{
James D. Chalmers ${ }^{1}$, Charlotte Balavoine ${ }^{2}$, Paola F. Castellotti ${ }^{3}$, \\ Christian Hügel ${ }^{4}$, Annabelle Payet ${ }^{5}$, Dennis Wat ${ }^{6}$ and Gernot Rohde $\mathbb{1}^{4}$
}

Affiliations: ${ }^{1}$ School of Medicine, University of Dundee, Dundee, UK. ${ }^{2}$ Chru Hôpitaux De Tours, Hôpital Bretonneau, Tours, France. ${ }^{3}$ ASST Great Metropolitan Hospital Niguarda, Milan, Italy. ${ }^{4}$ University Hospital Frankfurt, Frankfurt, Germany. ${ }^{5}$ Pneumologie, Centre Hospitalier Universitaire de la Réunion, Saint Pierre, Réunion. 'biverpool Heart and Chest Hospital, Liverpool, UK.

Correspondence: James D. Chalmers, University of Dundee, Nethergate, Dundee, DD1 4HN, Scotland, UK. E-mail: j.chalmers@dundee.ac.uk

ABSTRACT Once overlooked, awareness of nontuberculous mycobacterial pulmonary disease (NTM$\mathrm{PD})$ is rapidly rising, in line with increasing prevalence worldwide. The European Respiratory Society (ERS) International Congress 2019, held in Madrid, Spain, provided a platform for invigorating discussions and exciting new research in the field. This article explores approaches being taken to combat NTM-PD with a focus not only on novel prevalence and risk factor data, but also on emerging antimicrobials and their routes of delivery, and other potential treatment options in early clinical development.

@ERSpublications

Emerging research provides an insight into future treatment approaches for nontuberculous mycobacterial pulmonary disease (NTM-PD) - a serious, chronic, infectious disease that often presents in patients with underlying respiratory disease https://bit.ly/31spZpE

Cite this article as: Chalmers JD, Balavoine C, Castellotti PF, et al. European Respiratory Society International Congress, Madrid, 2019: nontuberculous mycobacterial pulmonary disease highlights. ERJ Open Res 2020; 6: 00317-2020 [https://doi.org/10.1183/23120541.00317-2020].

Received: 26 May 2020 | Accepted after revision: 3 Aug 2020

Copyright $\odot$ ERS 2020. This article is open access and distributed under the terms of the Creative Commons Attribution Non-Commercial Licence 4.0. 


\section{Introduction}

Nontuberculous mycobacterial pulmonary disease (NTM-PD) has a devastating impact on patients [1], with a poor long-term outcome. Global estimates of 5-year all-cause mortality are high, with estimates ranging from $25 \%$ to $40 \%$, and is not substantially improved by current treatment [2-5]. The condition typically occurs as a comorbid infection in patients with underlying respiratory disease, such as COPD, bronchiectasis and cystic fibrosis (CF) [6-8]. Patients often face a lengthy time to diagnosis, or their disease may be misdiagnosed as symptoms which can appear to be part of the underlying lung disorder rather than a separate infection [9]. The heterogeneity of presentation and underlying pulmonary disease means that care for patients with NTM-PD is often fragmented.

NTM-PD is steadily increasing in prevalence worldwide [10] and while awareness is rising, it remains suboptimal. The evidence base for NTM-PD investigation and management is limited compared with other respiratory disorders. In line with the increasing prevalence, however, there is renewed interest in NTM-PD and increasing research activity. This was reflected at the 2019 European Respiratory Society (ERS) Congress in Madrid, Spain, where several high-profile symposia and abstract sessions focussing on NTM-PD were scheduled. Sessions addressed the clinical characteristics of the disease, its incidence and prevalence, its management (including guideline-based treatment (GBT) and treatment outcomes) and potential new therapies on the horizon for this debilitating disorder. Here we present some of the highlights and novel data presented at the ERS Congress in 2019.

\section{Approach to evaluating the presence of NTM-PD at ERS 2019}

In order to explore NTM-PD content at the ERS 2019 Congress a committee chaired by Professors Chalmers and Rohde was convened. A review of the ERS 2019 Congress programme identified 110 sessions (abstracts and posters, symposia and speaker sessions) covering NTM-PD. Of the 110 identified sessions 24 (13 thematic posters; seven poster discussions; and four oral presentations) were prioritised by Professors Chalmers and Rohde for attendance by a committee member and then further review by all authors. Prioritised sessions covered the topics of diagnosis, prevalence, risk factors and treatment. Each NTM-PD session was presented to the committee for discussion and a selection for further inclusion in a publication was agreed. Given the nature of abstracts and symposia, content was reviewed subjectively, and those considered most interesting or robust were included.

\section{Epidemiology and microbiology}

Determining and understanding the causative agent for infection in NTM-PD can be difficult, as a result of widely varying epidemiological characteristics, considerable heterogeneity in the bacteria involved, and regional variations in species prevalence [10-13]. Individuals at risk of NTM-PD are diverse and include patients who are immunosuppressed $[14,15]$ and those with underlying diseases $[16,17]$.

Many NTM-PD studies are retrospective or observational and may be based on microbiological isolates. Non-tuberculous mycobacteria (NTM) are frequent environmental contaminants; therefore, the presence of an isolate in sputum culture does not confirm the presence of active infection. The American Thoracic Society/Infectious Diseases Society of America (ATS/IDSA) consensus criteria, published in 2007, for active infection requires the presence of clinical, radiological and microbiological evidence of NTM-PD. Our understanding is insufficient to enable linking of isolate or genomic information to causative agents and their virulence in causing disease requiring treatment. Genomic speciation for NTM has been undertaken [18] and it has been confirmed that, of the $>200$ species, the most common disease-causing isolates are Mycobacterium avium complex (MAC), Mycobacterium kansasii and Mycobacterium abscessus complex; however, it remains unclear how these isolates correlate with disease. Recent studies from Europe have begun to investigate the link between individual isolates and clinical disease. VANDE WeYGAERDE et al. [19] demonstrated that MAC, Mycobacterium gordonae and Mycobacterium xenopi were the most frequently isolated species in Belgian centres. M. abscessus, Mycobacterium malmoense and MAC were most frequently associated with clinical disease. In another study, Mycobacterium intracellulare was the most frequently isolated species but isolation of bacteria did not correlate with active infection [20]. Data from the European Bronchiectasis Registry (EMBARC) explored speciation in active disease and identified MAC as the most widely isolated organism derived in patients, followed by M. abscessus, Mycobacterium fortuitum, M. kansasii and M. malmoense [21]. The results of these studies confirm the diversity of species isolated in different disease cohorts. The major challenge remains to identify when a species is causing progressive disease and requires treatment [21].

While MAC is commonly isolated, infection does not necessarily result in pulmonary disease or the need for treatment. However, two radiological patterns have been identified in patients with pulmonary disease caused by MAC, each with differing disease outcomes [22]: fibrocavitary disease is more common in men with chronic pulmonary disease, while nodular bronchiectatic disease is usually associated with better 
outcomes and is more frequently found in post-menopausal women. A more recent study has also demonstrated that the presence of cavitation, however limited or small, is associated with worse outcomes, even if the predominant presentation is nodular bronchiectatic disease [22, 23]. For this reason, cavitation is considered a strong indication for initiating treatment in NTM-PD. The collated understanding of organism speciation, patient phenotype and disease outcomes therefore continues to evolve.

Testing for NTM can be costly, and there is not necessarily a correlation between the presence of NTM in respiratory cultures and active infection. Identifying individuals with a higher potential risk of NTM infection could allow for more targeted testing and could be useful for patients, clinicians and healthcare systems. Across the studies presented at ERS 2019, key risk factors for isolation of NTM were identified as bronchiectasis [20, 21]; immunosuppression [24]; and older age and the presence of specific radiographic characteristics [25]. Inhaled corticosteroid use has also been suggested as a risk factor for development of NTM in patients with underlying pulmonary disease [14, 26, 27]. In the future, a thorough understanding of patient phenotypes will be essential, as there is no standardised approach to testing in Europe. Thresholds for who is tested differ between institutions, with some testing all patients annually, while others will test those who fit the criteria for clinical suspicion (low body mass index, female sex, persistent cough) or who have frequent exacerbations.

As previously mentioned, the identification of active NTM is based on the ATS/IDSA criteria published in 2007, requiring clinical symptoms, radiological changes and microbiological criteria [28]. Patients meeting diagnostic criteria for NTM should undergo treatment, rather than watchful waiting, but the risks posed by NTM infection to the individual patient and the burden of treatment must be considered; tools that can identify the patients who would benefit most from therapy are eagerly anticipated.

\section{Treatment options: optimising current approaches}

While the effect of NTM-PD on patients' lives can be chronic and devastating [29], the impact of treatment can also be punishing as therapy is lengthy, involves complicated dosing schedules and may result in toxic adverse events (AEs) [9]. Treatment of NTM-PD requires a combination of oral, parenteral and nebulised antibiotics for an extended duration (18-24 months and for at least 12 months after achievement of sputum negativity) [28-31]; this may lead to poor patient adherence. AEs resulting from therapy are common and are a key reason for early treatment cessation, which is associated with treatment failure [32]. In a study conducted across three centres in Belgium of patients with NTM-PD caused by MAC, treatment adherence was low [33]. Of 60 patients receiving at least three drugs including a macrolide (in accordance with ATS/IDSA 2007 recommendations), just over half (33 out of 60) completed 12 months of therapy. Approaches to improve adherence and patient outcomes have been attempted, and a retrospective study presented at ERS 2019 explored the potential of using high-dose drugs with a reduced frequency of treatment administration. In this small cohort study comparing daily versus twice weekly therapy, the burden of AEs was reduced in the patients receiving less frequent treatment, but the study was too small to draw firm conclusions [34]. Therapy three times per week is also accepted for mild MAC disease, with similar treatment outcomes to daily therapy. Therefore, further testing of twice weekly therapy is of interest to reduce treatment burden. There remains a need for less toxic, more effective antibiotic therapies that do not need to be administered intravenously [33], and an improved understanding of which patients should receive and will benefit most from therapy [35].

Guidelines for treating NTM-PD are available from the ATS/IDSA [28], with an update due for publication in 2020. The joint 2007 ATS/IDSA NTM guidelines [28] recommend a minimum evaluation for patients with suspected NTM of a chest radiograph or chest high-resolution computerised tomography scan, plus at least two separate expectorated sputum samples or positive culture results from at least one bronchial wash or lavage, and exclusion of tuberculosis and lung cancer. Newer guidelines from the British Thoracic Society are also in place [36], with specific guidelines for patients with CF associated NTM [37]. Treatment success with 2007 ATS/IDSA GBT is often suboptimal; success rates (defined as culture conversion) for M. abscessus are $25-60 \%$ [38]. Success rates for MAC infection are higher, ranging between roughly $50 \%$ and $90 \%$, but there is a relapse rate (defined as recurrence of MAC infection from the same MAC strain rather than reinfection by a different strain) of $4-12 \%$. Results of a survey in the USA also suggested that adherence to GBT by clinicians was low [39]. European data are comparable; treatment practices as of 2017 were highly heterogeneous, with only $13 \%$ of patients eligible for therapy receiving the regimen recommended by ATS/IDSA 2007 recommendations [40].

Even when treatment is undertaken in line with guidelines, success is not guaranteed. In a Turkish single-centre study conducted over 10 years [41], 889 patients with NTM-positive sputum samples were identified. Of these, 102 (11.47\%) patients received 2007 ATS/IDSA GBT with just 40 patients (39.21\%) achieving treatment success. Recurrent disease was apparent in three patients $(2.94 \%)$ and 24 patients (23.52\%) had ongoing disease. Although the definition of treatment success and antibiotic regimens were 
not clearly defined, these data highlight that treatment success with 2007 ATS/IDSA GBT is suboptimal, and support results of meta-analyses suggesting a success rate for treating MAC with GBT ranging from $32 \%$ to $65 \%$ [42]. For M. abscessus treatment, the success rate was $45.1 \%$ with significantly greater success being observed among patients infected with M. abscessus subspecies massiliense compared to those infected with subspecies abscessus (81.4\% versus 33.5\%) [43].

Interpreting treatment success data is difficult in the absence of prospective studies that explore treatment outcomes according to the duration of therapy. Current recommendations for treatment length are determined by clinical experience rather than evidence-based data and, as such, understanding how long to treat patients remains an unmet clinical need.

\section{Treatment options on the horizon}

Given the dual issues of adherence, from the perspective of both clinician and patient, and efficacy of available treatment options, there is an urgent need for new approaches and therapies for NTM-PD caused by MAC and M. abscessus.

Nitric oxide (NO) is a naturally produced gas that plays a role in host innate defence mechanisms against various microorganisms including mycobacteria [44]. An abstract presented at ERS 2019 investigated the role of nitric oxide in treatment of M. abscessus. CHAU et al. [45] performed an in vitro study in which perfusion of M. abscessus strains with nitric oxide in combination with clofazimine and amikacin resulted in a greater reduction in $M$. abscessus bacterial load, compared with nitric oxide or clofazimine alone. These data are interesting and offer a new approach to treatment, although it is unclear whether in vitro findings will translate to animal models or human studies. Encouraging data are emerging that suggest a role for nitric oxide in the treatment of $M$. abscessus infection. In one study, compassionate adjunctive inhaled nitric oxide in two CF patients with M. abscessus infection demonstrated significant improvements in bacterial load [46], an observation supported by further data from the same group in a prospective pilot study [47]. In this study, nine patients with CF and M. abscessus experienced increases in both forced expiratory volume in $1 \mathrm{~s}\left(\mathrm{FEV}_{1}\right)$ and 6-min walking distance $(6 \mathrm{MWD})$ results from baseline as well as reductions in bacterial load [47]. While this study was not powered for significance and no statistics were presented, and culture conversion was not achieved, three out of nine patients did experience at least one negative culture during the study, suggesting that future research of nitric oxide against M. abscessus is warranted. It is also unclear from current data how nitric oxide might impact on M. abscessus sequestered within biofilms or macrophages, and what effect it may have alone or in combination with amikacin or clofazimine. A generator of nitric oxide from ambient air is currently being explored commercially for the treatment of NTM-PD [48] and it will be interesting to see the potential impact of this therapy on the condition in the next few years.

Another study presented at ERS 2019 explored the combination of amikacin and clofazimine on MAC infection [49]. In this study, patients were treated with either rifampicin/ethambutol/macrolide $(n=25)$ or rifampicin/ethambutol/macrolide plus amikacin/clofazimine $(n=19)$. Whilst combination therapy of rifampicin/ethambutol/macrolide plus amikacin/clofazimine demonstrated a trend towards better microbiological cure rates than rifampicin/ethambutol/macrolide ( $74 \%$ versus $52 \%)$ this was not statistically significant $(\mathrm{p}=0.337)$. A trend towards a faster time to sputum conversion (15 weeks versus 18 weeks) was also observed. It will be interesting to see how these data translate to randomised controlled clinical studies, ideally with comparator arms of amikacin and clofazimine alone to determine the impact of the combination.

Long-term data were presented from the CONVERT (Amikacin Liposome Inhalation Suspension (ALIS) for Treatment-Refractory Lung Disease Caused by Mycobacterium avium Complex) study by GRIFFITH et al. [50], which follow on from the original study published in 2018 [51]. In the study, patients with confirmed amikacin-susceptible MAC received ALIS plus 2007 ATS/IDSA recommended GBT (n=224) or GBT alone $(\mathrm{n}=112)$. Culture conversion by month 6 was achieved by 65 out of 224 (29\%) patients treated with ALIS plus GBT compared with 10 out of 112 (9\%) patients receiving GBT alone [51]. The recently presented data demonstrated that culture conversion was sustainable (defined as culture negative for all cultures taken from conversion through to the end of treatment) and durable (defined as culture negative in the absence of treatment for 3 months). At the end of treatment (up to 12 months post-conversion), $80 \%$ (52 out of 65 ) of patients treated with ALIS plus GBT who had achieved culture conversion remained culture negative compared with $30 \%$ (three out of 10) receiving GBT alone [50]. 3 months post treatment, durability of response was $63 \%$ (41 out of 65 ) of patients who received ALIS plus GBT and $0 \%$ (0 out of 10) of patients who had received GBT alone, although no statistics were reported. Tolerability of treatment and side-effects for extended antibiotic therapy were comparable with those presented in the original study [51]. There were no differences between treatment arms in terms of change in 6MWD results from baseline to month 8 $(\mathrm{p}=0.84)$. The CONVERT study was conducted in patients with refractory NTM-PD; in future, it will be 
important to evaluate longer-term follow-up results to explore the durability of culture conversion and reinfection rates. The low response rates in the GBT arm also emphasise the importance and challenging nature of refractory MAC disease. It will be interesting to explore further the impact of ALIS in patients as a strategy to prevent the development of refractory disease and to study its efficacy in patients newly diagnosed with NTM-PD caused by MAC.

\section{Future avenues for study}

As NTM-PD gains more interest from the research community, some new and attractive avenues are opening. To optimise treatment and outcomes for patients with this condition, it will be valuable to understand the link between isolates, pathogenicity and clinical relevance through the design of prospective studies that explore organisms and the trajectory of disease for individuals. These studies could investigate the impact of isolates on disease progression using prognostic factors such as change in lung function, body mass index and radiological changes/deterioration. It will also be important to understand the impact of NTM-PD and its treatment on individual patients.

From a clinical trial perspective, end-points that have an impact on patients' lives, including functional outcomes that can be applied effectively in studies, are needed $[28,52,53]$. Traditionally, the 6MWD test has been used as a measure of functional status in NTM disease. However, distance walked seldom correlates with disease severity and even very sick patients may be able to reach normal distances in the allotted time, leading to suggestions by the US Food and Drug Administration that it is a poor functional outcome [54]. In some studies where microbiological and culture conversion outcomes have been good, similar changes in quality of life measures have not been seen [51]. Other functional outcomes that could be used include improved body mass index and weight gain, as well as improvements in quality of life demonstrated using rating scales such as the St George's Respiratory Questionnaire and the Quality of Life Questionnaire-Bronchiectasis for which an NTM-PD module has recently been published [55]. An appraisal of these and other new functional outcomes is long overdue to provide clinical end-points that move beyond culture conversion and focus on patients. A disease-specific patient-reported outcome measure for NTM would be of significant value.

Patient goals for treatment need to be considered. These are diverse and include parameters such as fatigue, cough or breathlessness; such end-points are individual to patients and may be difficult to extrapolate to the clinical study setting. Greater emphasis on functional outcomes and how these change with treatment in NTM disease is clearly needed. As such, it will be important to develop and validate quality of life measures that can discriminate meaningfully between treatment success and failure.

\section{Increasing understanding across Europe}

For many years the study of NTM within Europe was neglected with little understanding of the epidemiological perspective of NTM species. One of the first publications to attempt to study NTM across multiple European countries was conducted by НовFsLOот et al. [56] and reported heterogeneity of NTM species causing clinically relevant disease across Europe. Since then, a range of initiatives have sought to increase awareness of NTM epidemiology, impact and treatment including NTM-NET, an international network of clinicians and scientists to promote clinically oriented research in NTM, and the European Multicentre Bronchiectasis Audit and Research Collaboration (EMBARC), which operates a European NTM registry alongside the successful European Bronchiectasis Registry in partnership with NTM-NET. European educational and advocacy initiatives have been developed, leading to the first NTM workshop held in Vienna in 2019 and the World Bronchiectasis and NTM conference held in Washington DC in 2018 and due to take place in Barcelona in 2020. These events have brought together hundreds of interested clinicians and scientists to stimulate collaborations and improve practice. Despite European efforts to increase awareness of NTM and to improve treatment in line with guideline recommendations, there is still some way to go. Even in 2020, a survey by NTM-NET [57] has shown that patients with bronchiectasis (those at highest risk of contracting NTM $[58,59]$ ) are not being routinely tested for NTM; this includes patients being considered for macrolide monotherapy, despite strong recommendations from 2020 guidelines that NTM testing should be conducted before treatment initiation [60]. Importantly, among surveyed clinicians there was relatively high $(>60 \%)$ understanding of the mortality and morbidity burden of NTM. NTM-PD, as demonstrated by its presence at the ERS Congress in 2019, is now out of the shadows.

\section{Conclusions}

NTM disease is a chronic condition that is difficult to treat and devastating for patients. Individuals at particular risk of infection are those with underlying respiratory disease or who are immunocompromised. However, a patient phenotype to guide treatment decisions that balances risks and benefits is still needed. It is also likely that the true prevalence of NTM in patients with pulmonary disease is underestimated 
BOX 1 Key points for nontuberculous mycobacterial pulmonary disease (NTM-PD) at the ERS Congress in 2019

- Whilst risk factors for NTM-PD are increasingly being understood there remains a need to develop a patient phenotype in order to target patient testing and initiate treatment.

- Despite increasing recognition of NTM-PD, it is clear that adherence to treatment remains low and it is likely this remains a key driver of relatively low treatment success. New treatment options are required.

- On the horizon for Europe stands amikacin liposome inhalation suspension (ALIS), which demonstrated greater treatment success in patients with refractory Mycobacterium avium complex (MAC) when added to guideline-based treatment (GBT) compared with GBT alone (29\% versus $9 \%$ ] [51].

- Ongoing analyses demonstrate a sustained culture conversion on-treatment in 52 out of 65

ALIS-treated patients (80\%) compared with three out of 10 patients on GBT alone (30\%) [50].

- Culture conversion is also durable when treatment is removed; 63\% of ALIS-treated patients maintained culture conversion versus $0 \%$ in those treated with GBT only [50].

- The addition of amikacin and clofazimine to GBT holds promise in treating NTM-PD, with the potential for greater culture conversion and a faster time to conversion than GBT alone, although full randomised clinical trials are awaited.

given the lack of standard testing for NTM as part of routine microbiological investigations in patients with pulmonary disease. Therefore, further prospective studies of the prevalence of NTM in pulmonary diseases and the identification of further risk factors for colonisation or infection of the lung are necessary.

As current therapies for NTM-PD are punishing for patients and have suboptimal outcomes, new approaches and treatment options are required. At ERS 2019, strategies discussed included flexible dosing schedules, new drug combinations in the form of amikacin and clofazimine, and data for the inhaled delivery of ALIS (box 1).

It is encouraging to see the field of NTM-PD expanding, with increasing interest, research and new publications. It is clear that the battle against NTM will need to take several fronts, from science to clinical management and increased awareness. The hope is that future research and the data generated will translate into improved and optimised patient care.

Conflict of interest: J.D. Chalmers reports grants and personal fees from AstraZeneca and Boehringer Ingelheim, grants from Gilead Sciences, grants and personal fees from GlaxoSmithKline, grants from Grifols, grants and personal fees from Insmed, and personal fees from Chiesi, Novartis and Zambon, outside the submitted work. C. Balavoine has nothing to disclose. P.F. Castellotti has nothing to disclose. C. Hügel reports personal fees from Boehringer Ingelheim and PulmonX, and nonfinancial support from Mukoviszidose e.V., Gilead, Insmed and Novartis, outside the submitted work. A. Payet has nothing to disclose. D. Wat reports grants and personal fees from Chiesi and Insmed, and personal fees from GlaxoSmithKline, outside the submitted work. G. Rohde reports personal fees from Boehringer Ingelheim, Chiesi, Essex Pharma, GlaxoSmithKline, Grifols, Insmed, Merck Sharp \& Dohme, Novartis, Pfizer, Roche, Solvay and Vertex, outside the submitted work.

Support statement: Editorial support was provided by Highfield, Oxford, UK, sponsored by Insmed Incorporated. Funding information for this article has been deposited with the Crossref Funder Registry.

\section{References}

1 FDA. The voice of the patient. Non-tuberculous mycobacterial lung infection. Public meeting: October 15, 2015; Report date April 2016. www.fda.gov/media/96932/download Date last accessed: February 2020.

2 Andréjak C, Thomsen VØ, Johansen IS, et al. Nontuberculous pulmonary mycobacteriosis in Denmark: incidence and prognostic factors. Am J Respir Crit Care Med 2010; 181: 514-521.

3 Ito Y, Hirai T, Maekawa K, et al. Predictors of 5-year mortality in pulmonary Mycobacterium avium-intracellulare complex disease. Int J Tuberc Lung Dis 2012; 16: 408-414.

4 Novosad SA, Henkle E, Schafer S, et al. Mortality after respiratory isolation of nontuberculous mycobacteria. A comparison of patients who did and did not meet disease criteria. Ann Am Thorac Soc 2017; 14: 1112-1119.

5 Marras TK, Campitelli MA, Lu H, et al. Pulmonary nontuberculous mycobacteria-associated deaths, Ontario, Canada, 2001-2013. Emerging Infect Dis 2017; 23: 468-476.

6 Jones MM, Winthrop KL, Nelson SD, et al. Epidemiology of nontuberculous mycobacterial infections in the US Veterans Health Administration. PLoS One 2018; 13: e0197976.

7 Bonaiti G, Pesci A, Marruchella A, et al. Nontuberculous mycobacteria in noncystic fibrosis bronchiectasis. Biomed Res Int 2015; 2015: 197950.

8 Martiniano SL, Nick JA, Daley CL. Nontuberculous mycobacterial infections in cystic fibrosis. Thorac Surg Clin 2019; 29: 95-108.

9 Chalmers JD, Aksamit T, Carvalho ACC, et al. Non-tuberculous mycobacterial pulmonary infections. Pulmonology 2018; 24: 120-131.

10 Prevots DR, Marras TK. Epidemiology of human pulmonary infection with non-tuberculous mycobacteria: a review. Clin Chest Med 2015; 36: 13-34. 
11 Park SC, Kang MJ, Han CH, et al. Prevalence, incidence, and mortality of nontuberculous mycobacterial infection in Korea: a nationwide population-based study. BMC Pulm Med 2019; 19: 140.

12 Kaguthi G, Nduba V, Murithi W, et al. The incidence of non-tuberculous mycobacteria in infants in Korea. J Trop Med 2019; 2019: 1273235 .

13 Jeon D. Infection source and epidemiology of nontuberculous mycobacterial lung disease. Tuberc Respir Dis (Seoul) 2019; 82: 94-101.

14 Brode SK, Jamieson FB, Ng R, et al. Increased risk of mycobacterial infections associated with anti-rheumatic medications. Thorax 2015; 70: 677-682.

15 Henkle E, Winthrop K. Nontuberculous mycobacteria infections in immunosuppressed hosts. Clin Chest Med 2015; 36: 91-99.

16 Marras TK, Campitelli MA, Kwong JC, et al. Risk of nontuberculous mycobacterial pulmonary disease with obstructive lung disease. Eur Respir J 2016; 48: 928-931.

17 Shteinberg M, Stein N, Adir Y, et al. Prevalence, risk factors and prognosis of nontuberculous mycobacteria infection among people with bronchiectasis: a population survey. Eur Respir J 2018; 51: 1702469.

18 Matsumoto $\mathrm{Y}$, Kinjo T, Motooka D, et al. Comprehensive subspecies identification of 175 nontuberculous mycobacteria species based on 7547 genomic profiles. Emerg Microbes Infect 2019; 8: 1043-1053.

19 Vande Weygaerde Y, Cardinaels N, Bomans P, et al. Clinical relevance of pulmonary non-tuberculous mycobacterial isolates in three reference centres in Belgium: a multicentre retrospective analysis. European Respiratory Journal 2019; 54: Suppl. 63, PA2158.

20 Lopez CM, Gallego CL, Rio Ramirez MT, et al. Nontuberculous mycobacteria, a 5-year study of respiratory tract samples. Eur Respir J 2019; 54: Suppl. 63, PA2941.

21 Ringshausen FC, Chalmers JD, Polverino E, et al. New isolation of non-tuberculous mycobacteria in patients with bronchiectasis - data from the European Bronchiectasis Registry (EMBARC). Eur Respir J 2019; 54: Suppl. 63 , PA2937.

22 Loebinger MR. Mycobacterium avium complex infection: phenotypes and outcomes. Eur Respir J 2017; 50: 1701380.

23 Koh WJ, Moon SM, Kim SY, et al. Outcomes of Mycobacterium avium complex lung disease based on clinical phenotype. Eur Respir J 2017; 50: 1602503.

24 Oliviera TJM, Guerra D, Fragosa J, et al. Clinical features of respiratory nontuberculous mycobacteria - a four-year comparison by immunosuppression status. Eur Respir J 2019; 54: Suppl. 63, PA2928.

25 Shirai T, Furuuchi K, Morimoto K, et al. Clinical characteristics and outcomes of patients with Mycobacterium avium complex lung disease positive for Aspergillus precipitating antibody. Eur Respir J 2019; 54: Suppl. 63, PA2940.

26 Liu VX, Winthrop KL, Lu Y, et al. Association between inhaled corticosteroid use and pulmonary nontuberculous mycobacterial infection. Ann Am Thorac Soc 2018; 15: 1169-1176.

27 Brode SK, Campitelli MA, Kwong JC, et al. The risk of mycobacterial infections associated with inhaled corticosteroid use. Eur Respir J 2017; 50: 1700037.

28 Griffith DE, Aksamit T, Brown-Elliott BA, et al. An official ATS/IDSA statement: diagnosis, treatment, and prevention of nontuberculous mycobacterial diseases. Am J Respir Crit Care Med 2007; 175: 367-416.

29 Yeung MW, Khoo E, Brode SK, et al. Health-related quality of life, comorbidities and mortality in pulmonary nontuberculous mycobacterial infections: a systematic review. Respirology 2016; 21: 1015-1025.

30 Egelund EF, Fennelly KP, Peloquin CA. Medications and monitoring in nontuberculous mycobacteria infections. Clin Chest Med 2015; 36: 55-66.

31 Brown-Elliott B, Nash KA, Wallace RJ Jr. Antimicrobial susceptibility testing, drug resistance mechanisms and therapy of infections with nontuberculous mycobacteria. Clin Microbiol Rev 2012; 25: 545-582.

32 Balavoine C, Blan F-X, Lanotte $\mathrm{P}$, et al. Adverse events during treatment of nontuberculous mycobacterial lung disease: do they really matter? Eur Respir J 2018; 52: Suppl. 62, PA2664.

33 Cardinaels N, Vande Weygaerde Y, Bomans P, et al. Adherence to NTM treatment guidelines in three reference centers in Belgium. Eur Respir J 2019; 54: Suppl. 63, PA2934.

34 Matsumoto M, Masuda K, Kaneshiro K, et al. Switch effects from daily to intermittent treatment with a daily regimen dosage for Mycobacterium avium complex pulmonary disease. Eur Respir J 2019; 54: Suppl. 63, PA2156.

35 Henkle E, Aksamit T, Barker A, et al. Patient-centered research priorities for pulmonary nontuberculous mycobacteria (NTM) infection. Ann Am Thorac Soc 2016; 13: S379-S384.

36 Haworth CS, Banks J, Capstick T, et al. British Thoracic Society guidelines for the management of non-tuberculous mycobacterial pulmonary disease (NTM-PD). Thorax 2017; 71: 1-64.

37 Floto RA, Olivier KN, Saiman L, et al. US Cystic Fibrosis Foundation and European Cystic Fibrosis consensus recommendations for the management of non-tuberculous mycobacteria in individuals with cystic fibrosis. Thorax 2016; 71: i1-i22.

38 Wu ML, Aziz DB, Dartois V, et al. NTM drug discovery: status, gaps and the way forward. Drug Discov Today 2018; 23: 1502-1517.

39 Adjeman J, Olivier KN, Prevots DR. Epidemiology of pulmonary nontuberculous mycobacterial sputum positivity in patients with cystic fibrosis in the United States 2010-2014. Ann Am Thorac Soc 2014; 11: 9-16.

40 Van Ingen JJ, Wagner D, Gallagher J, et al. Poor adherence to management guidelines in nontuberculous mycobacterial pulmonary diseases. Eur Respir J 2017; 49: 1601855.

41 Babalik A, Kuyucu T, Kutluhan F, et al. Pulmonary non-tuberculous mycobacterial infection: 889 cases. European Respiratory Journal 2019; 54: Suppl. 63, PA2159.

42 Kwon YS, Koh WJ, Daley CL. Treatment of Mycobacterium avium complex pulmonary disease. Tuberc Respir Dis (Seoul) 2019; 82: 15-26.

43 Chen J, Zhao L, Mao Y, et al. Clinical efficacy and adverse events of antibiotics used to treat Mycobacterium abscessus pulmonary disease. Front Microbiol 2019; 10: 1977.

44 Yang CS, Yuk JM, Jo EK. The role of nitric oxide in mycobacterial infections. Immune Netw 2009; 9: 46-52.

45 Chau T, Blade K, Da Silva J, et al. High efficacy of high-dose nitric oxide and its synergistic effect with antibiotics against Mycobacterium abscessus. Eur Respir J 2019; 54: Suppl. 63, OA4950.

46 Yacoby-Bianu K, Gur M, Toukan Y, et al. Compassionate nitric oxide adjuvant treatment of persistent Mycobacterium infection in cystic fibrosis patients. Pedatr Infect Dis J 2018; 37: 336-338. 
Bentur L, Gur M, Ashkenazi M, et al. Pilot study to test inhaled nitric oxide in cystic fibrosis patients with refractory Mycobacterium abscessus lung infection. J Cyst Fibrosis 2020; 19: 225-231.

48 Beyond Air. Differentiated Nitric Oxide Technology, Generated from Ambient Air. www.beyondair.net/technology Date last accessed: February 2020

49 Zweijpfenning S, Kops S, Boeree M, et al. Treatment of severe Mycobacterium avium complex pulmonary disease with adjunctive amikacin and clofazimine versus standard regimen alone, a retrospective study. European Respiratory Journal 2019; 54: Suppl. 63, OA3821.

50 Griffith DE, Thomson R, Addrizzo-Harris D, et al. Durability of culture conversion in patients receiving Amikacin Liposome Inhalation Suspension (ALIS) for treatment-refractory Mycobacterium avium complex lung disease (MAC-LD) in the CONVERT study. European Respiratory Journal 2019; 54: Suppl. 63, OA4951.

51 Griffith DE, Eagle G, Thomson R, et al. Amikacin Liposome Inhalation Solution for treatment-refractory lung disease caused by Mycobacterium avium complex (CONVERT): a prospective, open-label, randomized study. Am J Respir Crit Care Med 2018; 198: 1559-1569.

52 Diel R, Ringshausen F, Richter E, et al. Microbiological and clinical outcomes of treating non-Mycobacterium avium complex nontuberculous mycobacterial pulmonary disease. Chest 2017; 152: 120-142.

53 Punekar TS, Riley JH, Lloyd E, et al. Systematic review of the association between exercise tests and patient reported outcomes in patients with chronic obstructive pulmonary disease. Int J Chron Obstruct Pulmon Dis 2017 12: 2487-2506

54 Sullivan E. Lessons learned from completed NTM lung disease trials and implications for future trials. FDA 2018. www.fda.gov/media/124061/download Date last accessed: February 2020.

55 Henkle E, Curtis JR, Chen L, et al. Comparative risks of chronic inhaled corticosteroids and macrolides for bronchiectasis. Eur Respir J 2019; 54: 1801896.

56 Hoefsloot W, van Ingen J, Andrejak C, et al. The geographic diversity of nontuberculous mycobacteria isolated from pulmonary samples. Eur Respir J 2013; 42: 1604-1613.

57 Aliberti S, Ruffo Codesca K, Gori A, et al. The Italian registry of pulmonary non-tuberculous mycobacteria IRENE: the study protocol. Multidiscip Respir Med 2018; 9: Suppl. 1, 3.

58 Wagner D, van Ingen J, van der Laan R, et al. Non-tuberculous mycobacterial lung disease in patients with bronchiectasis: perceived risk, severity and guideline adherence in a European physician survey. BMJ Open Respir Res 2020; 7: e000498.

59 Andréjak C, Nielsen R, Thomsen VØ, et al. Chronic respiratory disease, inhaled corticosteroids and risk of non-tuberculous mycobacteriosis. Thorax 2013; 68: 256-262.

60 Smith D, Du Rand IA, Addy C, et al. British Thoracic Society guidelines for the use of long-term macrolides in adults with respiratory disease. BMJ Open Respir Res 2020; 7: e000489. 